\title{
Electron Correlation Models for Optical Activity
}

Höhn, E. G.; O. E. Weigang, Jr.

Published in:

Journal of Chemical Physics

Link to article, DOI:

$10.1063 / 1.1668773$

Publication date:

1968

Document Version

Publisher's PDF, also known as Version of record

Link back to DTU Orbit

Citation (APA):

Höhn, E. G., \& O. E. Weigang, J. (1968). Electron Correlation Models for Optical Activity. Journal of Chemical Physics, 48(3), 1127-1137. https://doi.org/10.1063/1.1668773

\section{General rights}

Copyright and moral rights for the publications made accessible in the public portal are retained by the authors and/or other copyright owners and it is a condition of accessing publications that users recognise and abide by the legal requirements associated with these rights.

- Users may download and print one copy of any publication from the public portal for the purpose of private study or research.

- You may not further distribute the material or use it for any profit-making activity or commercial gain

- You may freely distribute the URL identifying the publication in the public portal

If you believe that this document breaches copyright please contact us providing details, and we will remove access to the work immediately and investigate your claim. 


\title{
Electron Correlation Models for Optical Activity
}

\author{
E. G. HöHN* \\ Richardson Chemical Laboratories, Depariment of Chemistry, Tulane University, New Orleans, Louisiana \\ AND \\ O. E. Wetgang, JR. \\ Chemistry Laboratory A, The Technical University of Denmark, Lyngby
}

(Received 12 June 1967)

\begin{abstract}
A two-system no-overlap model for rotatory strength is developed for electric-dipole forbidden as well as allowed transitions. General equations which allow for full utilization of symmetry in the chromophore and in the environment are obtained. The electron correlation terms are developed in full detail for an ${ }^{1} A_{2} \leftarrow^{1} A_{1}$ $\left(C_{2 v}\right)$ transition of a chromophore interacting with a nonpolar anisotropic perturber. It ensues that perturber anisotropy of polarizability makes substantial contributions even in such zero-order forbidden transitions. The correlation terms for a strong electric-dipole allowed transition $\left({ }^{1} A_{1} \leftarrow^{-1} A_{1}\right)$ of the same chromophore gives sector rules that are decidedly different. An additive calculational scheme for the $n-\pi^{*}$ transition rotatory strengths of ketones shows the anisotropy effect to substantially modify, indeed sometimes become larger than, the simple octant rule behavior which occurs for isotropic perturbers. Since agreement with experimental rotatory strengths is correct in magnitude and sign and follows closely the observed variations, it appears that electron correlation is an important if not dominant perturbation mechanism for nonpolar substituents. An experimental prototype of the incomplete-screening-of-nuclei perturbation is considered which suggests that such a one-electron mechanism gives an incorrect sign of rotatory strength for the ketone back octants. The significance of the absolute signs associated with the octant rules of other chromophores are discussed.
\end{abstract}

\section{INTRODUCTION}

A detailed theory of optically active transitions in molecules can assume one of two limiting forms. When the part of a molecule primarily responsible for absorption of light in a particular frequency range is dissymmetric without external perturbation, optical activity is implicit straightforward in the description of the chromophore transition. However, when a chromophore is symmetric except for external perturbation, the induced optical activity of the transition can be expressed in terms for the symmetric chromophore and its molecular environment. As electron exchange between a chromophore and its surroundings becomes appreciable, the chromophoric system is accordingly enlarged. Thus, the latter limiting case smoothly passes over to the first through charge transfer and appreciable overlap between chromophore and environment. There are many discussions in the literature on these classifications and the theory applicable to them, especially most recently concerning the intermediate forms. ${ }^{1-10}$

\footnotetext{
* Present address: Department of Chemistry and Chemical Engineering, University of Saskatchewan, Saskatoon, Canada

$\dagger$ Regular address: Department of Chemistry, Tulane University, New Orleans, La.

1 A. Moscowitz, Advan. Chem. Phys. 4, 67 (1962)

2 I. Tinoco, Jr., Advan. Chem. Phys. 4, 113 (1962).

${ }^{3}$ A. Moscowitz, K. Mislow, M. A. W. Glass, and C. Djerassi, J. Am. Chem. Soc. 84, 1945 (1962).

${ }^{4}$ S. F. Mason, Quart. Rev. (London) 17, 20 (1963).

E D. J. Caldwell and H. Eyring, Ann. Rev. Phys. Chem. 15, 281 (1964).

${ }^{6}$ A. D. Liehr, J. Phys. Chem. 68, 3629 (1964).

7 J. C. Tai and N. L. Allinger, J. Am. Chem. Soc. 88, 2179 (1966).

${ }^{8}$ G. Wagnière, J. Am. Chem. Soc. 88, 3937 (1966).

- Y.-H. Pao and D. P. Santry, J. Am. Chem. Soc. 88, 4157 (1966).

${ }^{10}$ T.-Y. Chang, J. Chem. Phys. 45, 625 (1966).
}

The purpose of this paper is to examine in some detail the most general quantal model of optical activity for the latter limiting case, the inherently symmetric chromophore. Such has been reviewed quite generally by Tinoco. ${ }^{2}$ And while a great number of expressions suitable for application to experience have arisen from this approach, ${ }^{11-16}$ there is little danger of having exhausted its usefulness.

Two distinguishable models within the single latter limit evolved at an early date, coupled oscillator ${ }^{11}$ and one-electron ${ }^{16}$ theories. At that time the general consensus obtained that coupled oscillator theory was appropriate for strong electric-dipole allowed transitions while one-electron theory was to be utilized for the very weak transitions.

More recently, a modernization and extension of the Kauzmann-Walter-Eyring formulation of one-electron theory ${ }^{17}$ for optical activity in ketones applied by Moscowitz to the semiempirical calculation of several rotatory strengths ${ }^{1}$ has been accepted generally as the best theoretical basis for the ketone octant rule. ${ }^{18} \mathrm{How}$ ever, criticism has been directed toward the magnitude of screening constants necessary to give agreement with

11 J. G. Kirkwood, J. Chem. Phys. 5, 479 (1937).

12 W. Moffitt, D. D. Fitts, and J. G. Kirkwood, Proc. Natl. Acad. Sci. U.S. 43, 723 (1957).

${ }^{13}$ I. Tinoco, Jr., J. Chem. Phys. 33, 1332 (1960); 34, 1067

(1961); I. Tinoco, Jr. and R. W. Woody, ibid. 32, 461 (1960)

${ }^{14} \mathrm{~J}$. A. Schellman and P. Oriel, J. Chem. Phys. 37, 2114 (1962).

15 J. A. Schellman, J. Chem. Phys. 44, 55 (1966).

${ }^{16}$ E. U. Condon, W. Altar, and H. Eyring, J. Chem. Phys. 5, $753(1937)$.

${ }^{17}$ W. J. Kauzmann, J. E. Walter, and H. Eyring, Chem. Rev. 26, 339 (1940).

${ }^{18} \mathrm{~W}$. Moffitt, R. B. Woodward, A. Moscowitz, W. Klyne, and C. Djerassi, J. Am. Chem. Soc. 83, 4013 (1961). 
observed rotatory strengths. ${ }^{19,20}$ Furthermore, Schell$\operatorname{man}^{15}$ has raised the question of whether a variation in rotatory strength sign by quadrants, rather than octants, is not to be expected from the nature of the perturbation. Though quadrants are supported by general symmetry arguments, the detailed calculations have decidedly given sign variation with each octant.

That coupled oscillator theory applied to the ${ }^{1} A_{2} \leftarrow^{1} A_{1}$ transition of ketones also predicts an octant rule behavior ${ }^{21}$ has not been as widely appreciated. Indeed, at least one quite detailed calculation on an unsaturated ketone shows just such terms as being dominant, ${ }^{22}$ denoting a primary interaction between the carbonyl group and its ethylenic perturber via electron correlation, rather than the incomplete screening of nuclei invoked for one-electron theory. With their excited states lying at only moderately higher energies, saturated alkyl perturbers will produce similar effects.

Moreover, it appears that any model predicting selection rules and detailed coupling mechanisms for "forbidden character" in optical activity ${ }^{23}$ is significantly dependent on the relative importance of coupled oscillator terms as compared to one-electron terms. ${ }^{24}$ Thus it is important to examine as generally as possible the suitability of electron correlation as a perturbation mechanism in ketones dissymmetrically substituted with nonpolar groups and to compare even its qualitative features to those of the one-electron theory. No $a$ priori reason can be given why the allowed component of a rotatory strength cannot accidentally (i.e., not through symmetry) vanish or at least become very small while the forbidden component persists and becomes relatively important. Some reliable basis for predicting when such a situation might obtain is highly desirable. A program such as outlined here may indicate the course to be pursued in more extensive calculations of broader application.

\section{GENERAL THEORY}

\section{Electric-Dipole Forbidden-Magnetic-Dipole Allowed Transitions}

The theory is founded on the Rosenfeld expression for rotatory strength of the chromophore transition $m \leftarrow 0$,

$$
R_{0 m}=-i\left\langle A_{0} B_{0}|\boldsymbol{u}| A_{m} B_{0}\right\rangle \cdot\left\langle A_{m} B_{0}|\mathrm{~m}| A_{0} B_{0}\right\rangle
$$

where the correct wavefunctions are expanded to the

${ }^{19}$ M. V. Vol'kenshtein and M. P. Kruchek, Opt. Spectry. 9, $243(1960)$.

${ }^{20}$ A. D. Liehr, Progress in Transition Metal Chemistry, R. L. Carlin, Ed. (Marcel Dekker, Inc., New York, 1965), Vol, 2.

${ }_{21}$ O. E. Weigang, Jr., and E. G. Höhn, J. Am. Chem. Soc. 88, 3673 (1966).

${ }_{22}$ A. Moscowitz, A. E. Hansen, L. S. Forster, and K. Rosenheck, Biopolymers, Symp. No. 1, 75 (1964).

${ }^{23}$ O. E. Weigang, Jr., J. Chem. Phys. 42, 2244 (1965) ; 43, 3609 (1965).

${ }_{24}$ E. G. Höhn and O. E. Weigang, Jr., to be published. first order of perturbation,

$$
\begin{aligned}
& \left.\left|A_{m} B_{0}\right\rangle=\mid A_{m} B_{0}\right) \\
& \left.\quad+\sum_{r} \sum_{s}\left(E_{m}-E_{r}-E_{s}\right)^{-1}\left(A_{r} B_{s}|V| A_{m} B_{0}\right) \mid A_{r} B_{s}\right)
\end{aligned}
$$

with $m=0$ for the expansion of the ground state wavefunction $\left|A_{0} B_{0}\right\rangle$. The basis set $\left.\mid A_{r} B_{s}\right)$ consists of the simple product of spectroscopic state real wavefunctions of chromophore, $A_{r}$, and perturber, $B_{\varepsilon}$. No electron exchange and negligible differential overlap between the chromophore and perturber are assumed. The perturbation $V$ is then the electrostatic energy of interaction between the chromophore and perturber charge distributions.

The transition dipole moment expressions for the composite system, when the zero-order chromophore transition is electric-dipole forbidden but magneticdipole allowed, are then ${ }^{25}$ :

$$
\begin{aligned}
\left\langle A_{0} B_{0}\right| \boldsymbol{u} & \left|A_{m} B_{0}\right\rangle \\
& =\sum_{i \neq 0}\left(-E_{i}\right)^{-1}\left(A_{i} B_{0}|V| A_{0} B_{0}\right) \mathbf{u}_{m i} \\
& +\sum_{k \neq m}\left(E_{m}-E_{k}\right)^{-1}\left(A_{k} B_{0}|V| A_{m} B_{0}\right) \boldsymbol{u}_{0 k} \\
& +\sum_{j}-\left(E_{m}+E_{j}\right)^{-1}\left(A_{m} B_{j}|V| A_{0} B_{0}\right) \boldsymbol{u}_{0 j} \\
& +\sum_{l}\left(E_{m}-E_{l}\right)^{-1}\left(A_{0} B_{l}|V| A_{m} B_{0}\right) \mathbf{u}_{0 l}
\end{aligned}
$$

for the electric transition moment and

$$
\left\langle A_{m} B_{0}|\mathrm{~m}| A_{0} B_{0}\right\rangle=\left(A_{m} B_{0}|\mathrm{~m}| A_{0} B_{0}\right)=\mathbf{m}_{m 0}
$$

for the magnetic transition moment, each to the lowestorder nonvanishing terms.

Here $m$ is the state of the chromophore which combines with its ground state to give an electric-dipole forbidden-magnetic-dipole allowed transition; $i$ and $k$ indicate only those chromophore states which combine with states $m$ and 0 , respectively, to give nonzero electric-dipole moments. Then $j$ and $l$ are only those states of the perturber system giving nonvanishing electric transition-dipole moments, which also can be mixed into the zero-order transition. That the electric and magnetic transition dipole moments on the right in Eqs. (3) and (4) are simply moments of the zero-order subsystems follows from the assumed lack of electron exchange and negligible overlap between them together with the orthogonality of state wavefunctions for a given system. The center of gravity of the chromophoric

${ }^{25}$ We have hesitated to remove from the first and second summations and display separately a "charge transfer" term ${ }^{26}$ which must be considered with caution in the light of comments by Hameka and Goodman. ${ }^{27}$

${ }^{20}$ Reference 5, p. 306.

${ }^{27}$ H. F. Hameka and L. Goodman, J. Chem. Phys. 42, 2305 
subsystem has been chosen as the origin for the evaluation of its magnetic-dipole moment.

This restriction of the perturbation treatment sum over states in turn specifies that part of the total electrostatic interaction between chromophore and perturber which can effect a significant type of transition moment borrowing. The perturbation matrix elements in Eq. (3) are equivalent to electrostatic interactions between certain "charge distributions," $\left(A_{0} A_{m}\right)$ and $\left(B_{0} B_{j}\right)$ for example. They could be evaluated with the exact expression for the coulombic potential between charge groups. But, besides requiring detailed electronic wavefunctions based on some computational model for the groups, no particular generality would obtain. Alternatively and perhaps preferably, the Coulombic potential is expanded in charge multipole moments for nonoverlapping charge distributions. For our purposes, the potential can be written $a^{28}$ :

$$
V=\sum_{i} e_{t} \Phi\left(\mathbf{R}+\mathbf{0}_{t}\right)
$$

where $\Phi\left(\mathbf{R}+\mathbf{e}_{t}\right)$ is the potential arising from the chromophore for an external point charge located at $\mathrm{R}+\boldsymbol{\varrho}_{t} . V$ can be expanded in a Taylor series for small $\mathbf{0}_{t}$ about R. $\Phi$ is itself another expansion:

$$
\Phi=\sum_{s} e_{s} \cdot\left|\mathbf{R}-\mathbf{r}_{z}\right|^{-1}
$$

in a Taylor series for small $\mathbf{r}_{\boldsymbol{s}}$ about $\mathbf{R}$. Final expressions for some typical terms are given in Table I. There $R=|\mathbf{R}|$ becomes the distance between the centers of gravity of perturber and chromophore charge distribution; $X, Y$, and $Z$ are the signed components of that distance. The sum over products of the electronic charge with powers of electron coordinates relative to the respective centers of gravity become charge $\left(\epsilon_{0}\right)$, dipole $(\mu)$, quadrupole $(\theta)$, and if necessary, higher multipole components of the respective charge distributions $\left.\mid A_{0} A_{m}\right)$, etc. These electron coordinates, $x, y$, and $z$ centered on the respective subsystems, have the same spatial orientation as $X, Y$, and $Z$.

A most important and desirable result is that symmetry arguments can be applied to the matrix elements that ensue. As they are a generalization of the transition dipoles considered above, they also reduce to transition multipoles of the subsystems. Schellman has demonstrated the technique in a most elegant fashion but with exclusive consideration of the one-electron theory perturbation by incomplete nuclear screening. ${ }^{29}$

\footnotetext{
${ }^{28}$ H. Margenau, Rev. Mod. Phys. 11, 1 (1939).

${ }^{2}$ Reference 15. It is worthwhile noting that Schellman's expansion of the interaction potential is a crystal-field-like development of the total environment potential field evaluated at the chromophore, in contrast to the inverted approach of Eq. (5). The symmetry arguments are unchanged, but a sufficiently detailed description of the field will often require evaluation of many high-order multipoles; an approach which replaces, however, the necessity for treating the perturbation by many subsystems additively, as proposed in our work.
}

\section{Electric-Dipole Allowed Transition}

If instead the $m \leftarrow 0$ chromophore transition is electric-dipole allowed, the composite system transition moments of interest will be

$$
\left\langle A_{0} B_{0}|\mathbf{u}| A_{m} B_{0}\right\rangle=\left(A_{0} B_{0}|\mathbf{u}| A_{m} B_{0}\right)=\boldsymbol{u}_{0 m}
$$

and

$$
\begin{aligned}
& \left\langle A_{m} B_{0}|\mathrm{~m}| A_{0} B_{0}\right\rangle=\mathrm{m}_{m 0} \\
& -\sum_{i \neq 0}\left(-E_{i}\right)^{-1}\left(A_{i} B_{0}|V| A_{0} B_{0}\right) \mathrm{m}_{i m} \\
& +\sum_{k \neq m}\left(E_{m}-E_{k}\right)^{-1}\left(A_{k} B_{0}|V| A_{m} B_{0}\right) \mathrm{m}_{k 0} \\
& -\sum_{j}-\left(E_{m}+E_{j}\right)^{-1}\left(A_{m} B_{j}|V| A_{0} B_{0}\right) \\
& \quad \times\left[\left(\pi i E_{j} / h c\right) \mathrm{R} \times \mathbf{u}_{j 0}+\mathrm{m}_{j 0}\right] \\
& +\sum_{l}\left(E_{m}-E_{l}\right)^{-1}\left(A_{0} B_{l}|V| A_{m} B_{0}\right) \\
& \quad \times\left[\left(\pi i E_{l} / h c\right) \mathrm{R} \times \mathbf{u}_{l 0}+\mathrm{m}_{l 0}\right]
\end{aligned}
$$

utilizing the relationship that $m_{i m}=-m_{m i}$.

There is again a restriction of the perturbation sums over states, this time to those states $i$ and $k$ yielding nonvanishing magnetic transition-dipole moments of the chromophore and to those states $j$ and $l$ yielding electric transition dipole moments for the perturber. The result will be a basically different multipole description of the chromophore-perturber electrostatic interaction effecting significant transition moment borrowing.

Both Eqs. (3) and (8) contain terms which, on the basis of the perturbation matrix elements, can be associated with one-electron theory. In each case these are the first two of the borrowed transition moments, moments borrowed from the higher states of the chromophore. The last two moments, moments borrowed from the states of the perturber, are mixed in by electron correlative effects. However, this partition does not carry over to the second order of perturbation. Thus, a variational treatment can be expected to show a localization of effective transition moment in the chromophore that arises to a degree from an electron correlation mechanism. ${ }^{9}$

It can be ascertained that the rotatory strength subsequently obtained from Eqs. (3) and (4) or (7) and (8) are, to the first order, independent of the convenient origin to which angular momentum has been referred, ${ }^{30}$ the center of gravity of the zero-order chromophore in every case. The Appendix contains the pertinent arguments.

${ }^{20}$ For a detailed discussion see: A. Moscowitz, Modern Quantum Chemistry, O. Sinanoğlu, Ed. (Academic Press Inc., New York, 1965), Pt. 3, p. 31. 
TABLE I. Expanded terms of Coulombic potential between two nonoverlapping charge distributions $A$ and $B$.

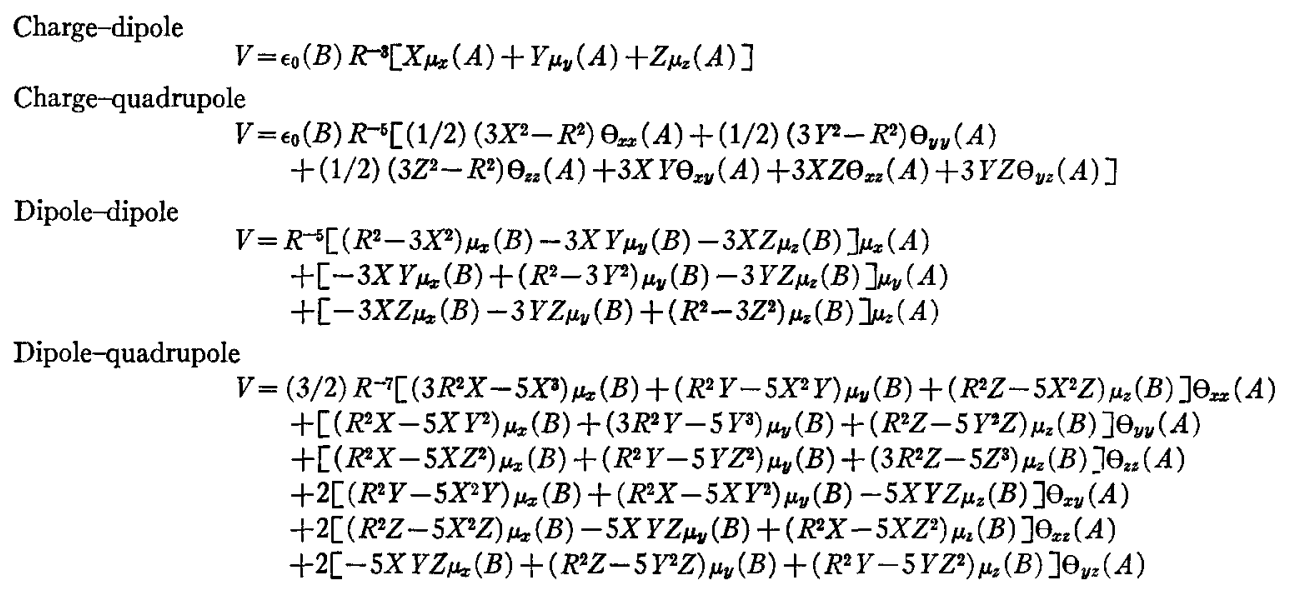

\section{${ }^{1} A_{2} \leftarrow{ }^{1} A_{1}\left(C_{2 v}\right)$ TRANSITIONS}

\section{Dynamic Coupling Terms ${ }^{31}$}

The techiques and equations derived so far are applicable in principle to chromophores of any point group. To demonstrate further reductions which are always possible, the expressions in Eqs. (3) and (4) must be referred to a specific case. ${ }^{1} A_{2} \leftarrow^{1} A_{1}$ transitions in the $C_{2 v}$ point group in particular have a wide range of applicability to optically active systems. The $n-\pi^{*}$ $(3000 \AA)$ transition of ketones is an obvious and even more particular example. Thus, certain conclusions of the most specific nature, that should not be applied indiscriminantely to other compounds, will be derived for this transition of the carbonyl chromophore and compared to the considerable body of empirical information available for ketones.

We refer now to the indexed states of Eq. (3), noting that $\mathrm{m}_{m 0}$ is $z$-axis polarized (see Fig. 1 ). Since then only $z$ components of the electric transition moments $\boldsymbol{u}_{i m}$, $\boldsymbol{u}_{0 k}, \boldsymbol{u}_{0 j}$, and $\boldsymbol{u}_{0 l}$ are important to the rotatory strength given in Eq. (1), the results of Table II are readily inferred. We have assumed groundstate wavefunctions totally symmetric for the point group of the subsystem, whether chromophore or perturber. When the perturber is taken to be nonpolar, ${ }^{32}$ such as a methyl or other alkyl group, the first nonvanishing terms of the expanded potential give the rotatory strength:

$$
\begin{aligned}
R_{0 m} & =6 i R^{-7} \sum_{l} E_{l}\left(E_{l^{2}}-E_{m}^{2}\right)^{-1}\left[\left(R^{2} Y-5 X^{2} Y\right) \mu_{0 l}{ }^{x}\right. \\
& \left.+\left(R^{2} X-5 X Y^{2}\right) \mu_{0 l}{ }^{y}-5 X Y Z \mu_{\mu_{0}}{ }^{z}\right] \mathrm{O}_{0 m^{x}}{ }^{x y} \mu_{0 l^{z}} m_{m 0^{z}}
\end{aligned}
$$

\footnotetext{
${ }^{31}$ We choose to use the names dynamic coupling and static coupling terms rather than coupled oscillator and one-electron terms. The latter often have been associated with particular, though not unique, formulations while the former, as will become apparent, are meant to be inclusive of diverse but fundamentally related effects. Note also that we use the term electron correlation in the sense of the exciton model rather than as an effect transcending Hartree-Fock self-consistency.

${ }^{32}$ We also assume throughout that the subsystems are not optically active.
}

for a coordinate system oriented as in Fig. 1. Equation (9) includes perturbation of the chromophore ground state as well as excited state; that is, the third and fourth summations of $\mathrm{Eq}$. (3) are brought to a single sum noting that $\left(A_{m} B_{l}|V| A_{0} B_{0}\right)=\left(A_{0} B_{l}|V| A_{m} B_{0}\right)$. The components of electric-dipole moment of $B$ (the perturber) are yet referred to coordinates with the same orientation as the carbonyl coordinate system.

Referring the perturber moments to a coordinate system appropriate to the symmetry of the perturber, each component of $\boldsymbol{u}_{0 l}$ is written in terms of components along new rotated coordinates (indicated here by primes) and the related direction cosines. ${ }^{33}$ The resulting expression is considerably simplified by the assumption of cylindrical symmetry, either actual or virtual through free rotation about an axis. With this averaging, particularly suitable for the concept of bonds as the source of dissymmetric perturbation, ${ }^{34}$ the rotatory strength is given by

$$
\begin{aligned}
R_{0 m}= & \frac{3}{2} i R^{-7}\left\{\left[-\left(R^{2} Y-5 X^{2} Y\right) \sin 2 \theta \cos \chi\right.\right. \\
& \left.+\left(R^{2} X-5 X Y^{2}\right) \sin 2 \theta \sin \chi\right] \beta\left(\nu_{0 m}\right) \bar{\alpha}\left(\nu_{0 m}\right) \\
& \left.-10 X Y Z\left[\alpha_{\| 1}\left(\nu_{0 m}\right) \cos ^{2} \theta+\alpha \perp\left(\nu_{0 m}\right) \sin ^{2} \theta\right]\right\} \theta_{0 m}{ }^{x} m_{m 0^{2}},
\end{aligned}
$$

where

$$
\begin{aligned}
& \alpha_{\| 1}=\alpha_{z^{\prime} z^{\prime}}=\left.\sum_{l} 2 E_{l}\left(E_{l}^{2}-E_{m}^{2}\right)^{-1}\left|\mu_{0 l^{2}}\right|^{\prime}\right|^{2}, \\
& \alpha_{\perp}=\alpha_{x^{\prime} x^{\prime}}=\alpha_{y^{\prime} y^{\prime}} ; \quad \bar{\alpha}=\frac{1}{3}\left(\alpha_{\mid 1}+2 \alpha_{\perp}\right),
\end{aligned}
$$

and $\beta\left(\nu_{0 m}\right)$ is a measure of the anisotropy of perturber polarizability ${ }^{11}$ :

$$
\beta=3\left(\alpha_{\|}-\alpha_{\perp}\right) /\left(\alpha_{\|}+2 \alpha_{\perp}\right),
$$

${ }^{33}$ For definition of the direction cosines as functions of the Eulerian Angles see E. B. Wilson, Jr., J. C. Decius, and P. C. Cross, Molecular Vibrations (McGraw Hill Book Co., New York, $1955)$, p. 285.

${ }^{34}$ For a clear discussion of the concept of bond interactions, see E. F. Haugh and J. O. Hirschfelder, J. Chem. Phys. 23, 1778 (1955). 
all measured at the frequency of the $m \longleftarrow 0$ chromophore transition.

When the perturbing group is isotropic such that $\alpha_{\| l}=\alpha_{\perp}=\bar{\alpha}$, the mean polarizability, Eq. (11) reduces to:

$$
R_{0 m}=-15 i R^{-7} X Y Z \bar{\alpha}\left(\nu_{0 m}\right) \theta_{0 m}^{x y} m_{m 0}{ }^{z} .
$$

A remarkable feature of this expression, noted earlier, 21,35 is that the correct absolute signs of the octant rule sectors for optically active ketones can be deduced from the Mulliken-McMurry model for the $n-\pi^{*}(3000 \AA)$ transition of the carbonyl group in ketones..$^{36}$ That is, $i \theta_{0 m}{ }^{x y} m_{m 0}{ }^{2}$ is real, negative, and large in atomic units.

Equation (10) gives two components for induced rotatory strength, that depending on a weighted mean polarizability and that depending on the polarizability anisotropy of the perturber. Figure 1 shows these component nodal surfaces, those locations for a perturber with respect to the carbonyl group where the component rotatory strength vanishes. The well-known octant rule surfaces obtain for the mean polarizability component.

The components dependent on polarizability anisotropy have nodal surfaces consisting of cones bisected by a single plane. The pronounced polarizability anisotropies evident from Kerr effects would seem to require that such components be included for a complete theory, contributing as they do on a scale equal to that for the mean polarizability component. With such consideration, anisotropic perturbers whose centers lie on octant rule surfaces can make sizeable contributions to rotatory strength. Indeed, anisotropic perturbers close to the simple octant rule surfaces can give contributions with signs reversed from those expected.

\section{Static Coupling Terms and One-Electron Theory}

In the case where the perturber is charged or polar, additional terms supplement those developed to this point. The first and second terms of Eq. (3) contribute then and, strictly speaking, in our model, only then to the rotatory strength. For a dipolar perturber group with the static moment $\boldsymbol{u}_{00}(\mathrm{~B})$ defining its $z^{\prime}$ axis, these additional terms reduce to:

$$
\begin{aligned}
& R_{0 m}{ }^{\mathrm{dip}}=-3 i R^{-7}\left[-\left(R^{2} Y-5 X^{2} Y\right) \sin \theta \cos \chi\right. \\
&\left.+\left(R^{2} X-5 X Y^{2}\right) \sin \theta \sin \chi-5 X Y Z \cos \theta\right] \mu_{00}{ }^{\prime}(\mathrm{B}) \\
& \times {\left[\sum_{i \neq 0}\left(-E_{i}\right)^{-1} \Theta_{0 i}{ }^{x y} \mu_{i m}^{2} m_{m 0}{ }^{z}\right.} \\
&\left.+\sum_{k \neq m}\left(E_{m}-E_{k}\right)^{-1} \Theta_{k m}{ }^{x y} \mu_{0 k} m_{m 0^{2}}\right] .
\end{aligned}
$$

Where the perturber carries a net charge $\epsilon_{0}(B)$,

${ }^{35}$ L. L. Jones, doctoral dissertation, University of Utah, 1961, gives a considerably more limited analysis and comparison of the coupled oscillator and traditional one-electron expressions.

${ }^{*}$ H. L. McMurry, J. Chem. Phys. 9, 231 (1941).
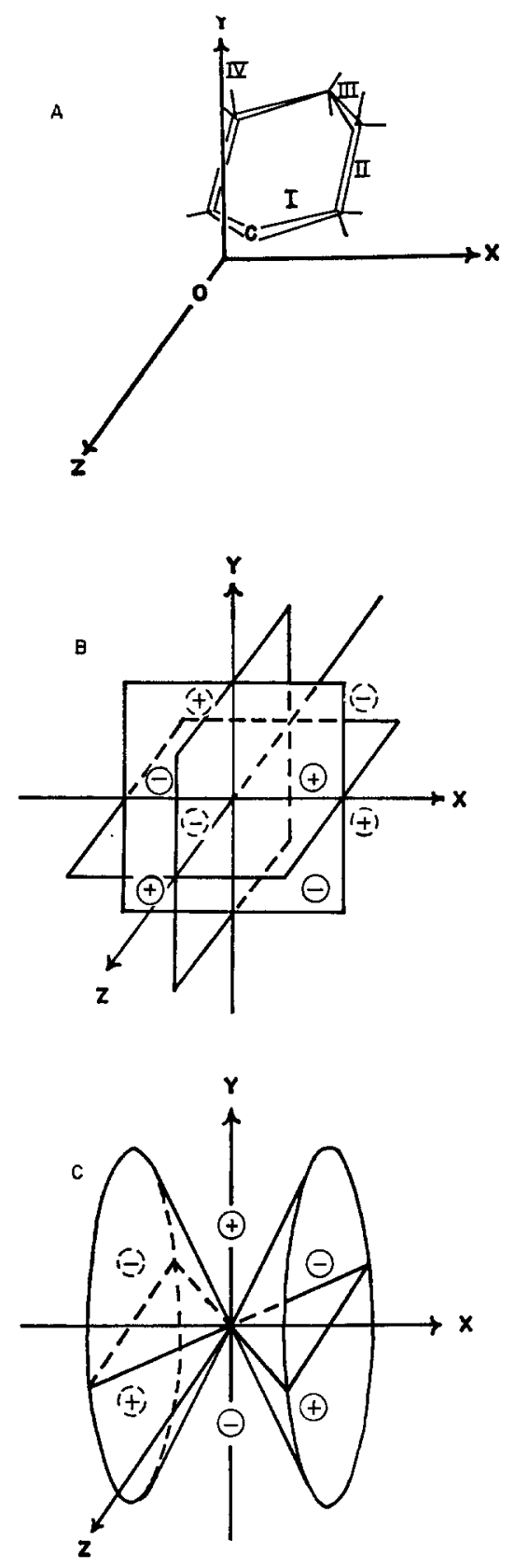

FIG. 1. (A) The coordinate system and perturber bond types. The bond types of the cyclohexanone ring in the chair form serve to indicate the possible angular orientations taken by bonds in substituted cyclohexanones with idealized geometry (Ref. 18) and methyl groups in the staggered conformation. (B) Perturbation nodal surfaces and absolute signs of rotatory strength for a ${ }^{1} A_{2} \leftarrow{ }^{1} A_{1} \quad\left(C_{2 v}\right)$ ketone transition under the influence of a dynamically coupled isotropic perturber ( $X Y Z$ dependence), from Eq. (10). (C) $X$-anisotropic nodal surfaces and absolute signs for the same transition and an anisotropic perturber, from first term in square brackets of Eq. (10), shown with signs for $\beta>0$ and $\sin 2 \theta \cos \chi>0$. [See Table IV for the values of $\sin 2 \theta \cos x$ and $\sin 2 \theta \sin \chi$ for the four bond types shown in (A).] The surfaces, with their conical axis along the $X$ axis, are pertinent for any perturber with a nonzero component of its optic axis along the $X$ axis. The intersections of the $X$-anisotropic nodal surfaces with the octant planes (b) are the lines $Y= \pm 2 X$ and $Z= \pm 2 X$. The $Y$-anisotropic nodal surfaces, associated with $\sin 2 \theta \sin \chi>0[\mathrm{Eq}$. (10) ] are obtained from a counterclockwise rotation of the surface by $\pi / 2$ around the $Z$ axis. 
TABLE II. The symmetry-restricted states of Eqs. (3) and (8).

Equation (3)

$\left.\begin{array}{cc}\text { State } & \text { Subsystem } \\ 0 & A \\ m & A \\ i & A \\ k & A \\ 0 & B \\ j & B \\ l & B\end{array}\right\}$

Equation (8)

$\left.\begin{array}{lc}\text { State } & \text { Subsystem } \\ 0 & A \\ m & A \\ i & A \\ k & A \\ 0 & B \\ j & B \\ k & B\end{array}\right\}$

Representation

$A_{1}$

$A_{2}$

$A_{2}$

$A_{1}$

Totally symmetric

\{ Varies in each of

various symmetries

$$
\begin{gathered}
\text { Basis function } \\
z \\
x y, m_{z}=(e / 2 m c)\left(x p_{y}-y p_{x}\right) \\
x y, m_{z}=(e / 2 m c)\left(x p_{y}-y p_{x}\right) \\
z
\end{gathered}
$$

Various components of the polar vector
Representation

$A_{1}$

$A_{1}$

$A_{2}$

$A_{2}$

Totally symmetric

Varies in each of

$\left\{\begin{array}{l}\text { varies in each of } \\ \text { various symmetries }\end{array}\right.$
Basis function

$$
\begin{gathered}
z \\
z \\
x y, m_{z} \\
x y, m_{z}
\end{gathered}
$$

Various components of polar vector

these terms are

$$
\begin{aligned}
R_{o m}{ }^{\mathrm{ohg}}=-3 i R^{-5} X Y \epsilon_{0}(B)\left[\sum_{i \neq 0}\left(-E_{i}\right)^{-1} \Theta_{0 i}{ }^{x y} \mu_{i m}^{z} m_{m 0}{ }^{z}\right. \\
\left.+\sum_{k \neq m}\left(E_{m}-E_{k}\right)^{-1} \Theta_{k m^{m}}{ }^{x y} \mu_{0 k}^{z} m_{m 0^{z}}\right] .
\end{aligned}
$$

Equation (15) has precisely the "quadrant rule" character of Schellman's analysis for a $C_{2 v}$ chromophore in a dissymmetric field. ${ }^{15}$ However, for the result to be applicable to optically active ketones requires the assumption of incomplete screening of the nuclei of uncharged nonpolar perturbers. Such a view is inconsistent with our uniform neglect of differential overlap between the subsystems.

In order to examine certain aspects of one-electron theory applied to ketones, we can relax this strict view and give an inverse exponential variation with distance to the parameter $\epsilon_{0}(B)$. Then a truncation of Eq. (15) that considers only one excited state $k$ compares closely to one-electron theory as it has been formulated previously for ketones. ${ }^{17,1}$ The pertinent penetration Coulomb integral has been expanded, of course, in multipoles of the subsystems, but its symmetry characteristics are preserved.

A resulting quadrant rule or octant rule for dissymmetric ketones is then related to the evaluation of $\theta_{k m}{ }^{x y}$. In the usual molecular-orbital treatment for the carbonyl group, $k$ is taken to be a state such that the matrix element is equivalent to $\Theta_{d x *} *^{x y}$ where ${ }^{1}$ (in our coordinate system)

$$
\begin{aligned}
\pi^{*} & =A 2 p_{y, 0}+B 2 p_{y, C}, \\
d & =C 3 d_{x z, 0}+D 3 d_{x z, C} .
\end{aligned}
$$

Neglecting two-center overlap (i.e., three-center terms in the penetration integral), the quadrupole moment $\theta_{d \pi} x_{y}$ vanishes. An octopole moment con- necting the two orbitals does not vanish and the further expansion of the potential will give $X Y Z$ (octant rule) dependence for the first nonvanishing terms. The resulting matrix element has been shown to be correct in sign. ${ }^{35}$ However, with the inclusion of reasonable overlap, $\theta_{d \pi *}{ }^{x y}$ gives an $X Y$-dependent component which must at some distance, apparently near $5 \AA$, become larger than the term containing $X Y Z$ dependence.

If $k$ is taken to be the $\pi \pi^{*}$ state, as suggested by Schellman, ${ }^{15}$ then the one-center contributions of the quadrupole $\theta_{n x^{x y}}$ are large in atomic units and the total moment is insensitive to a reasonable extent of overlap. The lower inverse power of $R$ and the proximity of the $\pi \pi^{*}$ state to the $n \pi *$ state would produce a very significant bias toward quadrant $(X Y)$ rule behavior. Yet many more appropriate states might be considered, and as well the perturbation of the chromophore ground state.

Thus, the one-electron formulation is ambiguous on the sector rule behavior, ${ }^{37}$ as is, unfortunately, much of the experimental data on the point. But it seems
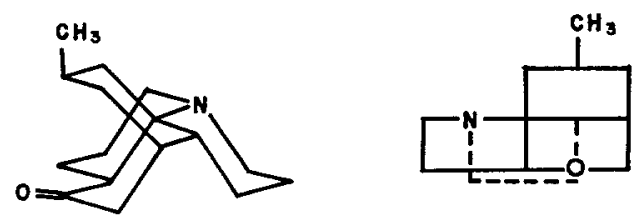

FIG. 2. The lycopodine molecule. See Footnote 38.

${ }^{37}$ It is the basic structure of the theory and a fortuitous circumstance of the carbonyl chromophore that the dynamic coupling does not have a similar ambiguity regarding absolute sign. The one-center quadrupole moments associated with $\Theta_{n \pi *}{ }^{x \nu}$ are large in atomic units and quite insensitive to inclusion of overlap while the sum of states is in this case absorbed in the perturber polarizability. Any developments of this nature for other chromophores must take heed of magnitude and sensitivity of the coupling parameters. 
possible to test its correctness of sign by empirically evaluating the entire sum over states. Equation (15) suggests that the effect of a real positive static charge is prototypic of the incomplete screening perturbation. The effect of a static dipole, though slightly more complex analytically, involves the same sum over states coefficient whose sign is to be determined.

What experimental evidence is available in fact argues that incomplete screening as a mechanism produces the incorrect sign of rotatory strength for the back octants of ketones. ${ }^{38}$ (Also see Fig. 2.) The interpretation is actually independent of a detailed analytical formulation; it assumes only the physical equivalence of the perturbations. The interpretation at the same time implies what finds support in some detailed calculation, ${ }^{22}$ that an electron correlation mechanism is dominant.

\section{${ }^{1} A_{1} \leftarrow^{1} A_{1}\left(C_{2 v}\right)$ TRANSITIONS \\ Dynamic Coupling Terms}

The expressions in Eqs. (7) and (8) are now applied to an electric-dipole allowed transition of the chromophore, polarized parallel to the $\mathrm{C}-\mathrm{O}$ direction ( $z$ axis). Again, one of the ketone transitions, the $\pi-\pi^{*}$, may be taken as an important example, though an assignment is less than certain. Table II contains the specifications of symmetry for significant contributions to rotatory strength, Eq. (1).

For nonpolar perturbers, the leading terms of the expanded potential give a rotatory strength ${ }^{41}$ :

$$
\begin{aligned}
& R_{0 m}=(\pi / h c) R^{-5} E_{m}\left[\frac{3}{2} Z\left(Y^{2}-X^{2}\right) \sin ^{2} \theta \sin ^{2} \chi\right. \\
& -\frac{1}{2} X\left(R^{2}-3 Z^{2}\right) \sin 2 \theta \sin \chi-\frac{1}{2} Y\left(R^{2}-3 Z^{2}\right) \sin 2 \theta \cos \chi \\
& \left.\quad-3 X Y Z \sin ^{2} \theta \cos 2 \chi\right] \beta\left(\nu_{0 m}\right) \bar{\alpha}\left(\nu_{0 m}\right)\left|\mu_{0 m}\right|^{2}, \quad(17)
\end{aligned}
$$

${ }_{38}$ The molecule lycopodine has been assigned the absolute configuration shown in Fig. 2 on the basis of a strong positive rotatory strength in dioxane and cyclohexane solvents. ${ }^{39}$ The perchlorate of lycopodine in methanol and water solutions gives a dichroism band (with nearly identical band shape for the former) about $\frac{1}{5}$ the magnitude and negative in sign. The addition of aqueous $\mathrm{HCl}$ to a methanol solution of the lycopodine free base causes a continuous decrease of rotatory strength from positive to negative values (without an obvious appearance of mixed-sign dichroism.bands).40 It seems most straightforward to relate the change in rotatory strength to the highly specific effect of the proton on the nitrogen. Consideration of less specific contributions would not seem to account for the observation. For example, a reasonably oriented ion-pair dipole could produce the observed effect only if the effect of a positive charge were also to decrease the rotatory strength. The effect related to the ion-pair polarizability would enhance ${ }^{18}$ rather than decrease the positive rotatory strength. Neither are assumptions of incorrect absolute configuration or conformational change with protonation without severe difficulties for a plausible explanation.

${ }^{2}$ W. A. Ayer, J. A. Berezowsky, and D. A. Law, Can. J. Chem. 41,649 (1963).

${ }^{40}$ We are indebted to Dr. Erik Larsen, H. C. Ørsted Institute, Copenhagen, for arranging for the determination of the circular dichroism, to Dr. Ayer for providing a sample of lycopodine and its perchlorate, and to Dr. Pierre Crabbé, Syntex, S. A., for bringing the work of Ayer et al. to our attention.

${ }_{41}$ The only contributing terms are taken to be from the explicit vector product terms of the third and fourth summations, based on the assumption that, in Kirkwood's' notation, $g(1)$ can be neglected in comparison to $g(0)$. Moffitt has commented on the relative magnitude of these terms. ${ }^{42}$ The same neglect holds for the case where $m_{m 0}$ does not vanish and its scalar product is formed with $\mathrm{Eq}$. (7) expanded as in $\mathrm{Eq}$. (3).

a W. Moffitt, J. Chem. Phys. 25, 467 (1956).
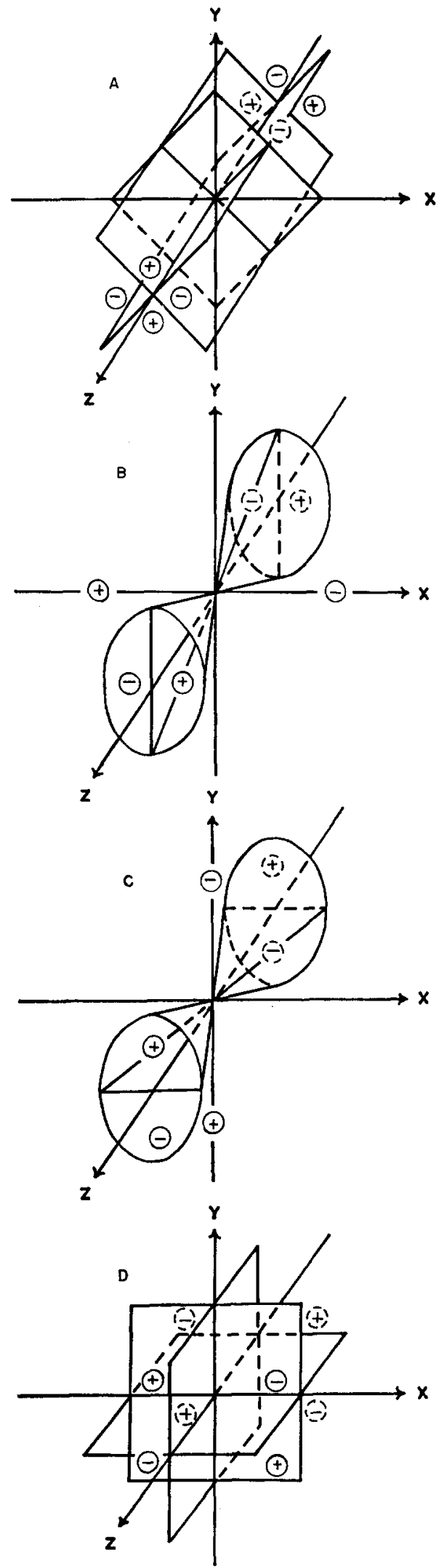

Fig. 3. Perturbation nodal surfaces for a ${ }^{1} A_{1} \leftarrow^{1} A_{1}\left(C_{2 v}\right)$ ketone transition under the influence of a dynamically coupled perturber. Signs are shown for $\beta$ and the trigonometric functions $>0$. (A), (B), (C), and (D) are obtained from the first, second, third, and fourth term in brackets of Eq. (17), respectively. 
TABLE III. Experimental and calculated rotatory strengths."

\begin{tabular}{|c|c|c|c|c|}
\hline Compound & $\begin{array}{l}\text { Observed } \\
R_{0 m} \times 10^{40}\end{array}$ & $\begin{array}{c}\text { Calculated } \\
\left(R_{0 m} / i \Theta_{0 m}^{x y} m_{m 0^{2}}\right) \\
\times 10^{-6}\end{array}$ & $\begin{array}{l}\rho \times 10^{46} \\
(\mathrm{obs} / \mathrm{calc}) \\
\times 10^{46}\end{array}$ & $R($ aniso $) / R($ total $) \mathbf{e}, \mathrm{d}$ \\
\hline 2,5-Dimethyl-cyclohexanone $(d i-e q .)^{\mathrm{e}}$ & +1.67 & $+0.81 \pm 0.00_{6}$ & 2.06 & $+0.240 \pm 0.012$ \\
\hline 2,2,5-Trimethyl-cyclohexanone $e^{e}$ & +6.21 & $+2.59 \pm 0.22$ & 2.40 & $-0.843 \pm 0.078$ \\
\hline trans-9-Methyl-1-decalone $e^{e}$ & +2.50 & $+1.26 \pm 0.21$ & 2.01 & $-1.437 \pm 0.269$ \\
\hline trans-9-Methyl-3-decalone $\mathrm{e}^{\mathrm{e}, \mathrm{f}}$ & -3.34 & -1.244 & 2.68 & -0.031 \\
\hline cis-9-Methyl-6-decalone (steroidlike) e, f, m & +0.676 & $\int+0.90$ & 0.752 & -0.473 \\
\hline cis-9-Methyl-6-decalone (nonsteroidlike) e, $\mathrm{f}, \mathrm{m}\}$ & & -3.22 & -0.210 & -0.166 \\
\hline $1 \beta, 9 \beta$-Dimethyl-trans-decalone- $3^{e}$ & -0.65 & $-0.43 \pm 0.00_{6}$ & 1.51 & $-0.357 \pm 0.043$ \\
\hline cis-10-Methyl-1-decalone $e^{e}$ & -0.686 & $-0.376 \pm 0.42^{\mathrm{b}}$ & 1.83 & $-6.965 \pm 8.686$ \\
\hline $8 \beta, 9 \beta$-Dimethyl-trans-decalone- $2^{\circ}$ & +4.73 & $+6.22 \pm 0.49$ & 0.76 & $-0.242 \pm 0.075$ \\
\hline Lycopodine $^{\mathrm{i}}$ & $+11.40^{\mathrm{j}}$ & +1.05 & 10.85 & -0.570 \\
\hline Dehydronorcamphor ${ }^{\mathbf{k}}$ & $+52.7^{j}$ & $+6.12^{\mathrm{n}}$ & 8.62 & $+0.246^{\mathrm{n}}$ \\
\hline Dimethyldibenzsuberone ${ }^{l}$ & $+95.3^{j}$ & $+12.27^{\mathrm{n}}$ & 7.77 & $-0.459^{\mathrm{n}}$ \\
\hline
\end{tabular}

a All entries are in cgs units.

${ }^{b}$ From Eq. (10), the average of staggered and eclipsed methyl group conformations as well as the corresponding deviation are given.

c $R$ (aniso), contribution from anisotropy-dependent part of Eq. (10); $R$ (total), from the entire equation.

d The average of this ratio for the staggered and eclipsed methyl conformations as well as the corresponding deviation are given.

e Reference 1, p. 97, and references cited therein. The observed values of reduced rotational strengths reported in Ref. 1 were converted into cgs units by multiplication with the proper factor.

${ }^{f}$ Reference 18, and references cited therein.

E C. Djerassi and W. Klyne, J. Chem. Soc. 1963, 2390.

h Value obtained for nonsteroidlike conformation.

i Reference 40 .

where polarizabilities [Eqs. (11) and (12)] and moments are all referred to convenient coordinates for the symmetry of the subsystems. Actual or virtual cylindrical symmetry of the perturbers has been assumed. Perturbers must be anisotropic to produce a rotatory strength that will be proportional to the ordinary absorption intensity of the zero-order chromophore transition. Equation (17) contains many of the features of Kirkwood's polarizability theory, ${ }^{11}$ but is stated in terms of a single rotatory strength. The nodal surfaces of each term in the brackets are shown in Fig. 3. Contrary to the case for symmetry deduced static coupling terms ${ }^{15}$ dynamic coupling does not at all give identical sector rules for electric-dipole forbidden and allowed transitions.

\section{Static Coupling Terms}

An additional perturbation due to static charge, or alternatively the mechanism of one-electron theory, can be developed in the manner illustrated above. It does not seem profitable to dwell on such at this time except to note the following. The effect of a static charge on the ${ }^{1} A_{1} \leftarrow^{1} A_{1}$ band varies simply as $X Y$, as Schellman's analysis ${ }^{15}$ shows, and can be expected to be in a range of magnitude similar to the effect on the ${ }^{1} A_{2} \leftarrow{ }^{1} A_{1}$ transition.

For the other allowed transitions of the $C_{2 v}$ point group, such an effect should be substantially larger, since charge-dipole rather than charge-quadrupole j From $\mathrm{CD}$ or ORD measurements, using the approximations given by C. Djerassi, in Oplical Rotatory Dispersion (McGraw-Hill Book Co., New York, 1960), Chap. 12, p. 165.

k K. Mislow and J. G. Berger, J. Am. Chem. Soc. 84, 1956 (1962). For the calculation, Gervais' coordinates were used (H. Gervais, thesis, Paris, 1966).

IE. L. Eliel, N. L. Allinger, S. J. Angyal, and G. A. Morrison, Conformational Analysis (John Wiley \& Sons, Inc., New York, 1965), p. 170. Each phenyl ring was considered as a single perturber, using the polarizability values 63.50 and 147.83 for $\alpha_{\|}$and $\alpha \perp$, respectively [Landolt-Börnstein, Zahlenwerte und Funktionen (Springer-Verlag, Berlin, 1951), Vol. 1, Pt. 3, pp. 509 et seq.].

In Footnote 47.

${ }^{n}$ Isotropic methyl groups assumed in this calculation.

coupling obtains in the perturbation matrix elements. Thus a possibly useful criterion for distinguishing between alternative spectral assignments of allowed bands is suggested.

\section{CALCULATIONS}

A limited computational program has been undertaken to compare experimental ketone rotatory strengths to those calculated with Eq. (10), the only expression for which available data justify such an effort. The consideration of perturber anisotropy, though certainly implicit in certain special calculations, has not received explicit attention in any generalized form. In just what manner it may modify a simpler octant rule is of considerable interest and importance.

Table III gives the results of calculations on ketones with nonpolar substituents. The molecules in the only extensive and reliable listing of molecular rotatory strengths ${ }^{1}$ have been utilized. Added to the table are three cases of special interest, even though the rotatory strengths may be known less reliably or the conditions of determination may not be comparable.

Since we have found that calculations on more "correct" geometries ${ }^{7,18}$ reveal little change in the features we wish to emphasize here, we have assumed idealized cyclohexanone geometry with tetrahedral angles where applicable (see Table IV). As a result, it is possible to read off $X, Y, Z$ coordinate values quickly from models without resorting to measurement (except, 
of course, in the cases of dehydronorcamphor and dimethyldibenzsuberone). Methyl groups were put in both the staggered and eclipsed conformations. The average and the deviation from average of these two calculations are reported. One may note there is often a significant dependence, in the case of cis-10-methyl-1decalone a total sign dependence, on such an apparently minor conformational feature. Carbon-carbon bond lengths were assumed to be $1.54 \AA$ and carbon-hydrogen were assumed to be $1.09 \AA$. The origin of the coordinate system was taken to be $0.6 \AA$ from the carbonyl carbon atom toward the oxygen atom.

Denbigh's values for components of the diagonalized polarizability tensors for $\mathrm{C}-\mathrm{C}, \mathrm{C}-\mathrm{C}$, and $\mathrm{C}-\mathrm{H}$ bonds, were used ${ }^{43}$ with a $10 \%$ increase to account for the dispersion of polarizability from static values. The polarizability values for the $\mathrm{C}-\mathrm{N}$ bond were taken from Le Fèvre and Le Fèvre. ${ }^{45}$

No attempt was made to calculate the zero-order observable and constant of the transition, $i \theta_{0 m}{ }^{x y} m_{m 0^{2}}$. Instead, the table lists for each molecule a semiempirical value, $\rho$, obtained from the calculation by comparison to the experimental rotatory strength. The latter was measured in a solvent, introducing a solvent field correction $f$, traditionally given by $\left(n^{2}+2\right) / 3 .{ }^{46}$

The average value of $\rho=\left(i \Theta_{0 m}{ }^{x y} m_{m 0}{ }^{z}\right) f$ from the data of Table III, excluding cis-9-methyl-6-decalone ${ }^{47}$ and the last three compounds, is $1.89 \times 10^{-46}$ cgs units. It would appear that an actual value must rather certainly lie in a range from 0.7 to $9.7 \times 10^{-46} \mathrm{cgs}$ units, based on the following considerations. The value of $i m_{m 0}{ }^{z}$ is rather reliably set at $0.9 \times 10^{-20}$ cgs units, ${ }^{48,49}$ though SCF MO calculations give only half that value. ${ }^{9} \mathrm{Calcu}-$

${ }^{43}$ K. G. Denbigh, Trans. Faraday Soc. 36, 936 (1940). Smith and Mortensen ${ }^{44}$ have criticized Denbigh's partition of molecular anisotropy of polarizability into bond values, contending the ratio of $>90$ for parallel to perpendicular polarizabilities of the $\mathrm{C}-\mathrm{C}$ bond is totally unrealistic. They show that only the value of $\Gamma_{\mathrm{CC}}=\beta_{\mathrm{CC}} \bar{\alpha}_{\mathrm{CC}}-2 \beta_{\mathrm{CH}} \bar{\alpha}_{\mathrm{CH}}$, for which they obtain a best value of 1.7 $\AA^{3}$, is directly obtainable from experiments on an homologous series of paraffin hydrocarbons. The ratio of $\mathrm{C}-\mathrm{C}$ bond polarizabilities would have to be reduced to less than 10 before it would sensibly change the value of $\beta_{\mathrm{CC}} \bar{\alpha}_{\mathrm{CC}}$ which is used in Eq. (10). But it is apparent that the partition of anisotropy is important since the bond polarizability values of Le Fèvre ${ }^{45}$ give decidedly more scattered values for $i \Theta_{0 m}{ }^{x} m_{m 0^{2}} f$ which range about a larger average value. As Denbigh's values give $\Gamma_{C C}$ equal to only $1.4_{5} \AA^{3}$, either the anisotropy of $\mathrm{C}-\mathrm{H}$ is lower or it has been given the wrong sign. Le Fèvre's values give $\Gamma_{C C}=0.7 \AA^{3}$ and the $\mathrm{C}-\mathrm{H}$ bond is considered isotropic, thus definitely underestimating the anisotropy.

${ }^{44}$ R. P. Smith and E. M. Mortensen, J. Chem. Phys. 32, 508 (1960).

${ }_{45}^{45}$ C. G. Le Fèvre and R. J. W. Le Fèvre, Chem. Ind. (London) 1955, 1121; J. Chem. Soc. 1956, 3459.

${ }^{46}$ O. E. Weigang, Jr., J. Chem. Phys. 41, 1435 (1964).

${ }^{47}$ For cis-9-methyl-6-decalone the steroidlike conformation is suggested by Moffitt $e t a l{ }^{18}$ as the most probable one. On the other hand, Eliel $e t$ al. (in Footnote l of Table III) discuss the possibility of both conformations occurring together in a nearly $1: 1$ ratio. From the results of our calculations it does seem likely that the observed value of -0.744 is a weighted mean of the values associated with the steroidlike and the nonsteroidlike conformation.

${ }^{48}$ Reference 1, p. 109.

${ }^{49}$ S. F. Mason, Mol. Phys. 5, 343 (1962).
TABLE IV. Euler angle functions of ideal cyclohexanone geometry.

\begin{tabular}{ccccc}
\hline Bond types $^{\mathrm{s}}$ & $\sin 2 \theta \cos \chi$ & $\sin 2 \theta \sin \chi$ & $\cos ^{2} \theta^{\mathrm{b}}$ & $\sin ^{2} \theta^{\mathrm{b}}$ \\
\hline I & 0.9428 & 0.0 & $\frac{1}{3}$ & $\frac{2}{3}$ \\
II & 0.0 & -0.9428 & $\frac{1}{3}$ & $\frac{2}{3}$ \\
III & -0.9428 & 0.0 & $\frac{1}{3}$ & $\frac{2}{3}$ \\
IV & 0.0 & 0.9428 & $\frac{1}{3}$ & $\frac{2}{3}$
\end{tabular}

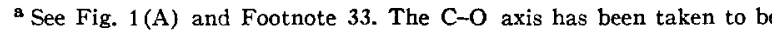
along the $z$ axis, the perturber bond axis along the $Z$ axis (in the notation of that reference).

b Note that by a fortuitous circumstance of the geometry, the mean polarizability can be factored from the $X Y Z$ term of $\mathrm{Eq}$. (10).

lated values of $\Theta_{0 m} x y$ may range from 0.6 to $8.1 \times 10^{-26}$ cgs units, the former value from SCF MO's, ${ }^{50}$ the latter value corresponding to the simple one-center $2 p_{x} \rightarrow 2 p_{y}$ hydrogen transition model that gives the correct experimental value for $i m_{m 0}{ }^{z}$. The value of $f$ according to the traditional Lorentz factor is about $\frac{4}{3}$.

Considering the uncertainties, other than computational they include solvent correction and molecular conformation, the results are as affirmative for the importance of dynamic coupling terms as one can expect. The correct absolute sign obtains, the order of magnitude is correct (even though the charge distributions are not very much smaller than the distances between their centers), the quite constant value of $\rho$ suggests the analytical expression of the octant rule is near correct. The calculational tests of one-electron theory certainly have been no more stringent.

Table III also gives the result of calculations on lycopodine, an unsaturated camphor derivative, ${ }^{22}$ and on dimethyl dibenzsuberone. ${ }^{51}$ The agreement with experiment is not the same as for the alkyl perturbed ketones though the order of magnitude is also acceptable. In fact, it is our opinion presently that actual solvent corrections are especially large for the saturated, less polarizable alkyl systems. ${ }^{52}$ Proper solvent corrections taking account of a dissymmetric solvent field, we believe, will go a long way to minimize the difference between these sets of data. But regardless, it is noteworthy that the electron correlation perturbation which by detailed calculation ${ }^{22}$ suffices to describe the optical activity of dehydronorcamphor is also adequate to describe the extremely large rotatory strength (and absorption intensity) of dimethyldibenzsuberone.

Table III also shows the degree to which the anisotropy terms have contributed to the respective calculated rotatory strengths. They are most often a small part of the algebraic total, either enhancing or decreasing the value for the isotropic terms. Three cases

50 Those of P. L. Goodfriend, F. W. Birss, and A. B. F. Duncan, Rev. Mod. Phys. 32, 307 (1960) were used. One-electron molecular orbitals from L. C. Cusachs' program at Tulane University yield nearly identical results.

${ }^{51}$ K. Mislow, M. A. W. Glass, A. Moscowitz, and C. Djerassi, J. Am. Chem. Soc. 83, 2771 (196i).

${ }^{52}$ O. E. Weigang, Jr. (to be published). 

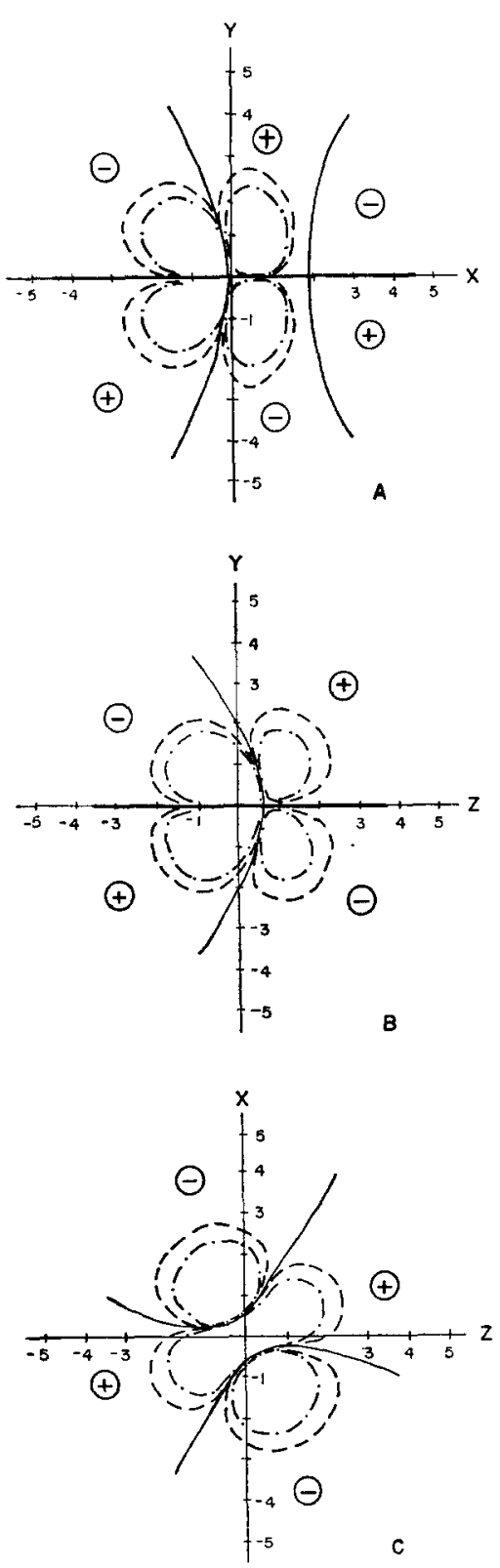

FIG. 4. Contours of rotatory strength for a dynamically coupled anisotropic perturber. $\beta$ is equal to 1.50 ; bond type $I$ is considered. (A) Parallel to $X Y$ plane at $Z=+1$. (B) Parallel to $Y Z$ plane at $X=+1$. (C) Parallel to $X Z$ plane at $Y=+1$.

are distinct exceptions. trans-9-Methyl-1-decalone and 2,2,5-trimethylcyclohexanone have ratios near unity and negative, denoting very significant anisotropy contributions against larger opposite signed isotropic contributions. cis-10-Methyl-1-decalone is unique for the eclipsed methyl conformer. For this conformer there is a positive ratio greater than unity showing an anisotropy term much larger than the isotropic term. The rotatory strength for the one conformer is thus in the main anisotropy dependent according to this calculation.
The cumulative effect of a dissymmetric array of bonds is thus quite complex as to whether anisotropy contributions tend to cancel or to reinforce. In these cases, anisotropy has not produced inversions from what is expected by the qualitative application of the octant rule (i.e., the isotropic term). An inversion from what has been predicted by qualitative application of the octant rule does occur with our calculation for the steroidlike form of cis-10-methyl-1-decalone. The qualitative prediction ${ }^{18}$ is that the steroidlike form is negative while our calculation gives a positive rotatory strength for the steroidlike form of the absolute configuration in Table III. However, there is no experimental evidence for a significant population of the steroidlike form, hence it is the nonsteroid conformation of the same absolute configuration which is reported in Table III.

To further define this anisotropy influence, Fig. 4 gives contours of net rotatory strength induced by a single nonpolar anisotropic bond at various positions with one of the four possible orientations of idealized cyclohexanone geometry (Fig. 1). The distortion of the ordinary octant rule surfaces is clearly discernible. In some planes, additional nodal surfaces have appeared.

\section{COMMENTS AND CONCLUSIONS}

Various chromophore point groups and transitions will generate greatly diverse routes in the reduction of the initial equation. Depending in part on the detailed electronic structure of the chromophore at hand, certain of the resulting expressions seem to produce unambiguous predictions regarding sector behavior and absolute sign. ${ }^{21}$ This is true especially when the first nonvanishing term of the potential expansion involves coupling transition multipoles that are large in atomic units. The expressions are most applicable to the lowest lying electronic transitions of the chromophore.

Even within the specific development of dynamic coupling for an ${ }^{1} A_{2} \leftarrow{ }^{1} A_{1}\left(C_{2 v}\right)$ rotatory strength, a variety of situations can arise in the qualitative aspect of predicting octant rule signs. If there is a simple molecular orbital description of the chromophore transition, the sign of $i \Theta_{0 m}^{x y} m_{m 0}{ }^{z}$ can usually be inferred from the one-center terms.

Robin et $a l^{53}$ have developed a molecular-orbital description of the cis-azoalkane system $n^{+} \rightarrow \pi^{*}$ $\left({ }^{1} B_{2} \leftarrow^{1} A_{1}\right)$ lowest spin-allowed transition from extensive calculations including configuration interaction. Though symmetry allowed, the calculated electricdipole transition moment is very small in atomic units. If it is so small that dipole-dipole coupling may be neglected, the rather large electric quadrupole and magnetic dipole moments expected give a development nearly identical to that for ${ }^{1} A_{2} \leftarrow{ }^{1} A_{1}$. An octant rule

${ }^{53}$ M. B. Robin, R. R. Hart, and N. A. Kuebler, J. Am. Chem. Soc. 89, 1564 (1967). 
with signs opposite those for ketones results when the $z$ coordinate lies along the $C_{2}$ symmetry axis and the molecule lies in the $x z$ plane.

The "mystery band" of the ethylene chromophore can be considered in the appropriate $D_{2 h}$ point group. The $\mathrm{CH} \rightarrow \pi^{*}$ "Berry assignment" sh4 should give an octant rule with signs the same as for ketones when the $z$ coordinate lies along the double bond and the molecule is in the $x z$ plane. On the other hand, the $\pi \rightarrow \mathrm{CH}^{*}$ "anti-Berry assignment" 55 is electric-dipole allowed out of plane and results in the anisotropy dependent analysis of Eq. (17) and Fig. 3. Even though the transition is only moderately strong, the dipole-dipole coupling in the latter assignment is here likely to persist as the dominant perturbation. This follows because the electric quadrupole moment of the transition vanishes strictly for this assignment, in contrast to the case for the cis-azoalkane transition.

It will be interesting, and of potential usefulness for assigning spectral transitions, to see whether such predictions are qualitatively correct. The basis, of course, is a quite simplistic model considering only onecenter terms and the assumption that dynamic coupling terms remain dominant for nonpolar perturbers of the chromophores.

The complications of the model seem scarcely more serious than those concerning neglect of differential overlap in the chromophoric system, incompleteness of basis set, and neglect of configuration interaction in more complete molecular orbital models. The generally stated additive perturbation expressions will more readily lend themselves to inclusion in the complex formulations of vibronic coupling and will more closely parallel the extant vibronic treatment of the formaldehyde ${ }^{1} A_{2} \leftarrow^{1} A_{1}$ transition. ${ }^{56}$

\section{ACKNOWLEDGMENTS}

This work was supported in part by a grant from the Petroleum Research Fund administered by the American Chemical Society. Grateful acknowledgement is hereby made to the donors of said fund. One of the authors (O.E.W.) wishes to acknowledge the hospitality of Chemistry Laboratory A, The Technical University, Denmark, during the tenure of a PRF-ACS International Award. The work was also supported in part by Public Health Service Grant GM-11644. The technical assistance of Miss Phyllis Trouard and Mr. Benes Trus in the computational program and a number of helpful discussions with Dr. L. C. Cusachs in connection with his evaluation of the ketone transition quadrupole moments is also gratefully noted.

${ }^{54}$ R. S. Berry, J. Chem. Phys. 38, 1934 (1963).

${ }^{65}$ M. B. Robin, R. R. Hart, and N. A. Kuebler, J. Chem. Phys. 44, 1803 (1966).

${ }^{66}$ J. A. Pople and J. W. Sidman, J. Chem. Phys. 27, 1270 (1957).

\section{APPENDIX: ORIGIN INDEPENDENCE OF ROTATORY-STRENGTH EQUATIONS}

On shifting by a constant vector $\mathbf{r}_{0}$ the coordinate origin to which the Rosenfeld expression is referred, Eq. (1), becomes

$$
\begin{aligned}
& R_{0 m}=-i\left\langle A_{0} B_{0}\left|\boldsymbol{u}^{\prime}\right| A_{m} B_{0}\right\rangle \cdot \mathbf{r}_{0} \\
& \times(e / 2 m c)(\hbar / i)\left\langle A_{m} B_{0}\left|\nabla^{\prime}\right| A_{0} B_{0}\right\rangle \\
&-i\left\langle A_{0} B_{0}\left|\mathbf{u}^{\prime}\right| A_{m} B_{0}\right\rangle\left\langle A_{m} B_{0}\left|\mathbf{m}^{\prime}\right| A_{0} B_{0}\right\rangle
\end{aligned}
$$

where primes indicate the new origin.

If only dynamic coupling terms are important, the first-order perturbation expression for the electricdipole moment, cf. Eq. (3), is generally

$$
\begin{aligned}
& \left\langle A_{0} B_{0}\left|\mathbf{u}^{\prime}\right| A_{m} B_{0}\right\rangle=\left(A_{0} B_{0}\left|\mathbf{u}^{\prime}\right| A_{m} B_{0}\right) \\
& \quad+\sum_{l} 2 E_{l}\left(E_{m}^{2}-E_{l}^{2}\right)^{-1} \\
& \times\left(A_{0} B_{0}|V| A_{m} B_{l}\right)\left(A_{0} B_{0}|\boldsymbol{u}| A_{0} B_{l}\right)
\end{aligned}
$$

while the corresponding dipole velocity is

$$
\begin{gathered}
\left\langle A_{0} B_{0}\left|\nabla^{\prime}\right| A_{m} B_{0}\right\rangle=\left(A_{0} B_{0}|\nabla| A_{m} B_{0}\right) \\
+\sum_{l} 2 E_{m}\left(E_{m}^{2}-E_{l}\right)^{-1} \\
\times\left(A_{0} B_{0}|V| A_{m} B_{l}\right)\left(A_{0} B_{0}|\nabla| A_{0} B_{l}\right)
\end{gathered}
$$

from the property that

$$
\left(A_{0} B_{0}|\nabla| A_{0} B_{l}\right)=-\left(A_{0} B_{l}|\nabla| A_{0} B_{0}\right) .
$$

Then

$\left(\hbar^{2} / m\right)\left\langle A_{0} B_{0}\left|\nabla^{\prime}\right| A_{m} B_{0}\right\rangle=E_{m}\left\langle A_{0} B_{0}\left|\mathbf{u}^{\prime}\right| A_{m} B_{0}\right\rangle$

is obtained with the relationship

$$
\left(\hbar^{2} / m\right)\left(A_{0} B_{0}|\nabla| A_{0} B_{l}\right)=E_{l}\left(A_{0} B_{0}|\mathfrak{u}| A_{0} B_{l}\right) .
$$

This is so since $\left(A_{0} B_{0}\right)$ and $\left.\mid A_{0} B_{l}\right)$ are exact wavefunctions for a Hamiltonian which is the sum of Hamiltonians for $A$ alone and $B$ alone.

The colinearity implied in Eq. (A4) for the two vectors as they are defined is a sufficient condition to ensure that the first term of Eq. (A1) vanishes.

For the static coupling terms no such colinearity of vectors can be implied from the first-order expressions. Nevertheless, for the case of chromophore transitions that are electric-dipole forbidden-magnetic-dipole allowed, the first term of Eq. (A1) vanishes to a firstorder approximation. Higher-order corrections could give contributions that become comparable to the second term only for magnitude of $\mathbf{r}_{0}$ near $100 \AA$. But for the case of static coupling chromophore transitions electric-dipole allowed, independence of origin cannot be as conveniently argued. It would obtain insofar as the first-order wavefunctions, Eq. (2), are exact solutions for some Hamiltonian. 